\title{
MODE-IMPEDANCE TECHNIQUE FOR MODELING OF ELECTROMAGNETIC WAVE PROPAGATION IN PLASMAS
}

\author{
A.G. Shalashov, E.D. Gospodchikov \\ Institute of Applied Physics RAS, Nizhniy Novgorod, Russia, \\ E-mail:ags@appl.sci-nnov.ru
}

The impedance technique, also known as the particular case of the invariant embedding approach, is generalized for modeling of electromagnetic wave propagation in the anisotropic and gyrotropic media with spatial dispersion such as hot magnetized plasmas. That provides a new way for analytical and numerical studies of "full wave" problems in plasma physics that require exact solutions of Maxwell equations. Coupling of electromagnetic and electrostatic plasma modes in electron cyclotron range is considered as an example of application of the proposed technique.

PACS 52.35.Hr, 41.20.Jb, 42.25.Bs, 52.50.Sw

\section{INTRODUCTION}

In the present communication we review a relatively simple general technique for modeling the propagation of electromagnetic waves in hot magnetized plasmas. Specific features of such media are complex (gyrotropic and anisotropic) dielectric response, spatial dispersion, and existence of several normal waves with different polarizations which may be coupled in spatially inhomogeneous plasma. The basic problem considered below is reconstruction of electromagnetic fields inside and outside of a stationary plasma layer highlighted by incident radiation. This problem primarily corresponds to the plasma heating by high frequency waves in magnetic traps. Mathematically such processes are naturally described by a boundary problem for Maxwell equations with inhomogeneous constitutive relations. However, there is a regular way to transform this problem to a purely evolutional problem defined only by initial conditions. In this form the problem becomes more convenient both for developing the analytical solutions and for numerical calculations.

The considered approach is a particular form of the more general method of invariant embedding [1]. In most cases the invariant embedding technique has been applied to a scalar wave equation [2]. However, wave propagation in magnetized plasmas is more adequately described by vector wave equations. In this case, a straightforward generalization of the standard invariant embedding technique is possible, but it involves essential technical difficulties, see e.g. [3]. The main goal of the present paper is demonstration of a new interpretation of the invariant embedding technique. A particular new development concerns the interaction between the normal waves of the studied medium in terms of the evolution of the reflection operator that couples counterpropagating normal waves. This reformulation of the invariant embedding approach gives rise to new evolutionary equations that are more transparent, physically intuitive, and very flexible for further analytical transformations or numerical studies.

The technique may be especially useful being applied to linear mode-conversion problems characterized by large evanescent regions the interacting modes. This makes straightforward numerical integration of the wave equation usually impossible, because the evanescent wave which propagates against the direction of integration is inevitably raised from a numerical noise resulting in an exponentially growing solution in the direction of integration. This exponentially growing part tends to suppress all real physics in the round-off errors long before the integration procedure reached the other end of the domain. The similar troubles exist near the plasma resonances characterized by quite different wavelengths and damping lengths for the "fast" and "slow" waves. The mode-impedance technique provides a way of a numerical treatment of stiff wave problems which requires much less computer resources than usual (finite element or FDTD) methods, for more details see [4].

\section{MODE-IMPEDANCE TECHNIQUE FOR MAXWELL EQUATIONS}

Let us consider a plane monochromatic wave which is incident to plasma layer inhomogeneous along the coordinate $z$ and limited within the range $a \leq z \leq b$. Generalization over a three-dimensional geometry may be found in [4]. Outside the layer there is some "external" medium which is assumed to be homogeneous. Our final goal is to find the wave distribution inside the slab and the reflection and transmission matrixes characterizing the wave distribution outside the slab. To do so, one must solve Maxwell equations inside the slab,

$$
\operatorname{rot} \mathbf{E}=i k_{0} \mathbf{H}, \quad \operatorname{rot} \mathbf{H}=-i k_{0} \bar{\varepsilon}(z) \mathbf{E},
$$

with proper boundary conditions - fixed incident wave at the one end and absence of the ingoing (incident) wave at the other end. Here $k_{0}=\omega / c$ is the vacuum wave vector, and the plasma dielectric response is defined by $3 \times 3$ tensor operator which contains derivatives over the $Z$ coordinate in case of spatial dispersion

$$
\widehat{\varepsilon}(z)=\widehat{\varepsilon}_{0}\left(z, k_{x}\right)+\widehat{\varepsilon}_{1}\left(z, k_{x}\right) \partial_{z}+\widehat{\varepsilon}_{2}\left(z, k_{x}\right) \partial_{z}^{2}+\ldots
$$

Let us introduce the mode-impedance technique. At first step, one must choose the minimum set of field variables that describe a particular problem, and convert Maxwell equations to the following form:

$$
\partial_{z} \boldsymbol{\Psi}=\hat{M} \boldsymbol{\Psi},
$$

where $\hat{M}(z)$ is a matrix of scalar functions containing no differential operators, and $\boldsymbol{\Psi}(z)$ is a field vector like

$$
\boldsymbol{\Psi}=\left(E_{x}, H_{x}, E_{y}, H_{y}, E_{z}, H_{z}, \partial_{z} E_{x}, \partial_{z} E_{y}, \partial_{z} E_{z}, \partial_{z}^{2} E_{x} \ldots\right) .
$$


Some derivatives of field components may be also included as components of $\boldsymbol{\Psi}$-vector to preserve form (1) in cases with spatial dispersion, for example, related to electrostatic waves. Here the derivatives are treated as independent field variables what allows to take into account higher dimensionality due to spatial dispersion

Once $\boldsymbol{\Psi}$ and corresponding $\hat{M}$ are known, one can specify the boundary conditions. The most natural way is to find the mode composition of the ingoing and outgoing radiation. Indeed, outside the slab one may assume that $\boldsymbol{\Psi} \propto \exp \left(i k_{z} z\right)$, then equation (1) reduces to an algebraic problem $i k_{z} \boldsymbol{\Psi}_{0}=\hat{M}(a) \boldsymbol{\Psi}_{0}$. Eigenvectors $\boldsymbol{\Psi}_{0}$ give polarizations of the ingoing and outgoing waves propagating outside the slab; wave vectors $k_{z}$ may be defined as roots of the corresponding dispersion relation,

$$
\operatorname{det}\left(\widehat{M}_{0}-i k_{z} \hat{I}\right)=0 \text {. }
$$

Normal modes may be sorted into two groups. Let $\boldsymbol{\Psi}_{01}^{+}, \boldsymbol{\Psi}_{02}^{+}, \ldots \boldsymbol{\Psi}_{0 n}^{+}$denote all modes propagating or evanescent in the positive direction towards the $z$ axis $\left(\operatorname{Re} k_{z}>0\right.$ or $\left.\operatorname{Re} k_{z}=0, \operatorname{Im} k_{z}>0\right)$; and $\boldsymbol{\Psi}_{01}^{-}, \boldsymbol{\Psi}_{02}^{-}, \ldots \boldsymbol{\Psi}_{0 n}^{-}$ denote all modes propagating or evanescent opposite to the $z$ axis (either $\operatorname{Re} k_{z}<0$ or $\operatorname{Re} k_{z}=0, \operatorname{Im} k_{z}<0$ ). In most cases the set of eigenmodes forms a basis for $\boldsymbol{\Psi}$ vector - at any point the wave field may be decomposed over the normal waves of the external medium

$$
\boldsymbol{\Psi}(\mathrm{z})=\sum_{i=1}^{n} \mathrm{E}^{+}(\mathrm{z}) \boldsymbol{\Psi}_{0 i}^{+}+\sum_{i=1}^{n} \mathrm{E}^{-}(\mathrm{z}) \boldsymbol{\Psi}_{0 i}^{-} .
$$

Here $E_{i}^{ \pm}(z)$ are $2 n$ unknown functions that will be used as new field variables; obviously their number is equal to the dimension of the initial $\boldsymbol{\Psi}$-vector. Let us group these functions into two new $n$-dimensional vector fields:

$$
\varepsilon^{+}=\left(\mathrm{E}_{1}^{+}, \mathrm{E}_{2}^{+}, \ldots \mathrm{E}_{n}^{+}\right), \varepsilon^{-}=\left(\mathrm{E}_{1}^{-}, \mathrm{E}_{2}^{-}, \ldots \mathrm{E}_{n}^{-}\right) .
$$

By definition, the components of these vectors give the amplitudes of normal modes with definite propagation direction in the external medium outside the slab. After decomposition of the wave field, Maxwell equation may be formally rewritten as equations for two coupled counterpropagating waves

$$
\left\{\begin{array}{c}
\partial_{z} \varepsilon^{+}=\widehat{t}^{+} \varepsilon^{+}+\widehat{r}^{-} \varepsilon^{-} \\
-\partial_{z} \varepsilon^{-}=\widehat{t}^{-} \varepsilon^{-}+\widehat{r}^{+} \varepsilon^{+}
\end{array} .\right.
$$

which should be solved in the range $a<z<b$ with boundary conditions, e.g. $\varepsilon^{+}(a)=\varepsilon^{\text {inc }}, \quad \varepsilon^{-}(b)=0$. Matrix coefficients in (3) may be obtained from

$\left(\begin{array}{cc}+\widehat{t}^{+} & +\widehat{r}^{-} \\ -\widehat{r}^{+} & -\hat{t}^{-}\end{array}\right)=\widehat{U}^{-1} \hat{M} \hat{U}, \quad \widehat{U}=\left(\boldsymbol{\Psi}_{01}^{+}, \ldots \boldsymbol{\Psi}_{0 n}^{+}, \boldsymbol{\Psi}_{01}^{-}, \ldots \boldsymbol{\Psi}_{0 n}^{-}\right)$,

$\widehat{U}$ is a matrix with columns formed by vectors $\boldsymbol{\Psi}_{0 i}^{ \pm}$.

Boundary problem (3) may be further reduced to evolutional problem defined only by initial conditions. Let us formally introduce "mode-impedance" matrix that couples forward and backward waves as

$$
\mathcal{E}^{-}(z)=\widehat{R}(z) \mathcal{E}^{+}(z) \text {. }
$$

Note, that this unknown yet operator $\hat{R}(z)$ may be considered as the reflection matrix of the reduced layer $[z, b]$ for the wave incident from $z<a$. In particular,
$\widehat{R}(b)=0$ since there is no reflection from a "zero" layer. Substituting (4) into equations (3), one may exclude $\varepsilon^{+}$ and obtain the following matrix equation:

$$
-\partial_{z} \hat{R}=\hat{R} \hat{r}^{-} \hat{R}+\hat{R} \hat{t}^{+}+\hat{t}^{-} \hat{R}+\hat{r}^{+}, \quad \hat{R}(b)=0 .
$$

In case of one-dimensional inhomogeneity, the evolutional equation is reduced to a set of nonlinear ordinary differential equations for components of the impedance matrix $\hat{R}(z)$, which can be easily integrated numerically for arbitrary distribution of the dielectric tensor in space. The integration should start from the right boundary $z=b$ with zero initial condition. Once $\hat{R}(z)$ is known, the wave field distribution inside the slab may be retrieved as well as the reflected and transmitted waves outside the slab (for a given incident wave). Indeed, the forward wave $\varepsilon^{+}(z)$ may be obtained by integrating the following equation from the boundary $z=a$ :

$$
\partial_{z} \varepsilon^{+}=\left(\hat{t}^{+}+\widehat{r}^{-} \widehat{R}\right) \varepsilon^{+}, \quad \varepsilon^{+}(a)=\varepsilon^{\mathrm{inc}} .
$$

Finally the backward wave $\mathcal{E}^{-}$may be retrieved from (4).

\section{TEST WAVE PROBLEM}

The proposed formalism has been proved to be very effective in modeling of wave propagation in inhomogeneous magnetized plasmas. To provide a working example we consider electromagnetic waves propagating in dense plasmas in electron cyclotron frequency range. We focus our attention on the problem of linear coupling of the electromagnetic modes in the inhomogeneous plasma, subsequent excitation of the electrostatic electron Bernstein mode by the extraordinary mode and cyclotron damping of these waves. This problem has many applications in ionosphere and astrophysics; in recent years interest in linear mode coupling in this frequency range has increased appreciably in connection with the high-frequency plasma heating in spherical tokamaks and optimized stellarators [5].

For simplicity we assume that the ambient magnetic field is directed along the $x$ axis, and the hot plasma dielectric response is modeled by [4]:

$$
\widehat{\varepsilon}(z)=\left(\begin{array}{ccc}
\varepsilon_{\|}+\chi_{1} k_{0}^{-2} \partial_{z}^{2} & i \xi k_{0}^{-1} \partial_{z} & \xi k_{0}^{-1} \partial_{z} \\
-i \xi k_{0}^{-1} \partial_{z} & \varepsilon_{\perp}+\chi_{2} k_{0}^{-2} \partial_{z}^{2} & i g-i \chi_{3} k_{0}^{-2} \partial_{z}^{2} \\
\xi k_{0}^{-1} \partial_{z} & -i g+i \chi_{3} k_{0}^{-2} \partial_{z}^{2} & \varepsilon_{\perp}+\chi_{4} k_{0}^{-2} \partial_{z}^{2}
\end{array}\right) .
$$

Here $\varepsilon_{\|}=1-\omega_{p e}^{2} / \omega^{2}, \varepsilon_{\perp}=1-\omega_{p e}^{2} /\left(\omega^{2}-\omega_{c e}^{2}\right)$, and $g=$ $\omega_{p e}^{2} \omega_{c e} /\left(\omega^{2}-\omega_{c e}^{2}\right) \omega$ are usual parameters of cold plasma; $\xi, \chi_{1}, \ldots \chi_{4}$ are resulted from the thermal motion of plasma electrons, explicit expressions for these coefficients may be found in [4]. Following the general algorithm we chose $\boldsymbol{\Psi}=\left(E_{x}, H_{x}, E_{y}, H_{y}, E_{z}, \partial_{z} E_{z}\right)$, corresponding matrix $\hat{M}$ and propagating modes $\boldsymbol{\Psi}_{0 i}^{ \pm}$are found in [4]. We use Mathematica ${ }^{\mathrm{tm}}$ software both for deriving the final equations (5), (6) in analytical form and for subsequent numerical integration. From a physical point of view, the six components of $\boldsymbol{\Psi}$-vector correspond to three modes - the ordinary (O), extraordinary $(\mathrm{X})$ and Bernstein $(\mathrm{B})$ waves, propagating in each direction. So, six roots of the dispersion 
relation (3) must be attributed to the $\mathrm{O}, \mathrm{X}$ and $\mathrm{B}$ modes. Note that all three modes should exist in the external medium, thus we must specify very rarefied plasma outside the slab.

We simulate an oblique propagation of waves in hot dense plasma with a humped density profile:

$$
n_{e} \propto\left[1-(\mathrm{z} / L)^{4}\right]^{16}, T_{e} \sim 1 \mathrm{keV}, \omega_{p e}, \omega_{c e}>\omega .
$$

It is well known that both $\mathrm{O}$ and $\mathrm{X}$ modes could not propagate towards dense enough plasma when launched from vacuum [5]. However, being launched at a specific angle, the $\mathrm{O}$ mode couples effectively to the slow $\mathrm{X}$ mode behind the upper hybrid resonance layer [6]. In this case one should expect the strong absorption instead of reflection from the layer of dense plasma due to further conversion of the slow $\mathrm{X}$ mode to the electrostatic $\mathrm{B}$ mode, which freely propagates towards the plasma density increase and is absorbed at the nearest electron cyclotron harmonics. In the Figure the absorption for the incident $\mathrm{O}$ mode is plotted versus the launching angle.

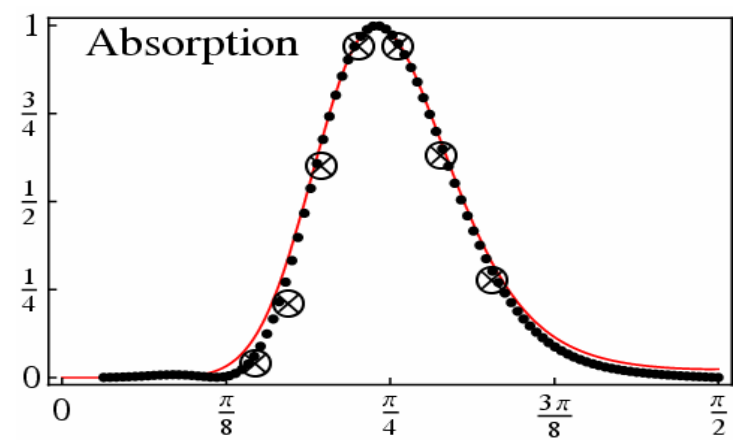

Absorption coefficient for the incident $O$ mode versus launching angle. $O$ mode cutoff corresponds to

$k_{0} L_{n}=4.8, \omega_{c e} / \omega=0.88, T_{e}=1 \mathrm{keV}$ and $\vartheta_{\mathrm{opt}}=43^{\circ}$
One can see the perfect coincidence of the well-known analytical result by Mjølhus [6] (thin line) and the results of the mode-impedance approach both in cold (dots) and hot (crosses) plasmas. Thus, we demonstrate a direct full wave justification of the widely used analytical approach.

\section{CONCLUSIONS}

The boundary problem for Maxwell equations is reduced to two consecutive nonlinear initial problems (5) and (6). Note that the only heuristic action in this technique is formulation of a model for the dielectric response (what selects the studied modes) and choice of proper components of vector $\boldsymbol{\Psi}$. Although the derived analytical expressions typically are rather lengthy, numerical integration of the evolutionary equations remains stable even for the most difficult cases involving several very different scale-lengths (in particular, we avoid uncontrolled exponential growth of evanescent modes which may result in instability of numerical integration). In summary, by performing all analytical transformations using a computer, we develop a flexible and fast tool for studying full wave problems in complex media. More applications may be found in [4].

\section{REFERENCES}

1. M.R. Scott. Invariant Imbedding and its Applications to Ordinary Differential Equations. London:“AW”, 1973.

2. V.I. Klyatskin. Invariant Embedding Method in Wave Propagation Theory. Moscow: "Nauka", 1986.

3. K.G. Budden. Lectures on Magnetoionic Theory. London: "Blackie", 1964.

4. A.G. Shalashov, E.D. Gospodchikov // Plasma Physics Control. Fusion. 2010, v. 52, N 2, p. 025007.

5. H.P. Laqua // Plasma Phys. Control. Fusion. 2007, v. 49, p. R1-R42.

6. E. Mjølhus // J. Plasma Phys. 1984, v. 31, p. 7.

Article received 13.09.10

\section{МЕТОД МОДОВОГО ИМПЕДАНСА ДЛЯ ЗАДАЧ РАСПРОСТРАНЕНИЯ ЭЛЕКТРОМАГНИТНЫХ ВОЛН В ПЛАЗМЕ}

\section{А.Г. Шалашов, Е.Д. Господчиков}

Предложена удобная и физически прозрачная формулировка метода инвариантного погружения для решения задач распространения электромагнитных волн в средах с анизотропным и гиротропным диэлектрическим откликом с пространственной дисперсией, в частности, в горячей магнитоактивной плазме. Новый метод может быть использован для аналитических и численных расчетов “полноволновых" проблем физики плазмы, требующих решения полной системы уравнений Максвелла. В качестве примера такого приложения рассмотрена проблема взаимодействия электромагнитных и электростатических плазменных мод в электронном циклотронном диапазоне частот.

\section{МЕТОД МОДОВОГО ІМПЕДАНСУ ДЛЯ ЗАДАЧ ПОШИРЕННЯ ЕЛЕКТРОМАГНІТНИХ ХВИЛЬ В ПЛАЗМІ}

\section{А.Г. Шалашов, С. Д. Господчиков}

Запропоновано зручне та фізично прозоре формулювання методу інваріантного занурення для вирішення завдань поширення електромагнітних хвиль у середовищах 3 анізотропним та гіротропним діелектричним відгуком $з$ просторовою дисперсією, зокрема, у гарячій магнітоактивній плазмі. Новий метод може бути використаний для аналітичних та чисельних розрахунків “повнохвильових" проблем фізики плазми, що потребують вирішення повної системи рівнянь Максвелла. Як приклад такого застосування розглянуто проблему взаємодії електромагнітних та електростатичних плазмових мод в електронному циклотроному діапазоні частот. 